\title{
Small fibre dysfunction in Hypothyroidism - a prospective study using methods of small fibre function and structure
}

\author{
S Sharma, G Rayman \\ Diabetes Research Unit, The Ipswich Hospital NHS Trust, Ipswich, Suffolk, United Kingdom
}

Correspondence: sanjeeev.sharma@ipswichhospital.nhs.uk

\section{Introduction}

- Neurologic complications, including polyneuropathy, are well-known findings in hypothyroidism (HypoT), with a prevalence ranging from 42 to $72 \%$

- In neurologic series of polyneuropathy, the prevalence of HypoT is reported to be around $2-4 \%$. However, there is paucity of information regarding the prevalence and clinical course of small fibre neuropathy in HypoT

- Recent studies have demonstrated reduced intra-epidermal small nerve fibre density in both subclinical and overt HypoT reversed by levothyroxine (LT4) treatment

- The Laser doppler imager flare (LDI FLARE$_{\text {) }}$ technique (Figure 1a \& 1b) is a simple noninvasive technique, involving skin heating and measuring the size of the axon-reflex mediated flare using laser Doppler imagery has been shown to be a sensitive marker of C-fibre dysfunction in early diabetes and impaired glucose tolerance patients. It has excellent correlation with intra-epidermal nerve fibre density

- Corneal confocal microscopy (CCM) can identify early small nerve fibre structural damage and has been shown to accurately quantify the severity of diabetic neuropathy (Figure 2)

Aims

- This prospective study examines small fibre function (SFF) and structure (SFS) in a mixed cohort of HypoT subjects before and after LT4 treatment

$>$ before and after LT4 treatment

$>$ and compares the outcomes with a cohort of healthy controls $(\mathrm{HC})$

Methods

- SFF and SFS was determined in 20 patients with HypoT (TSH $\geq 35$ miu/L) - 15 primary and 5 post-radioiodine - along with 20 agematched $\mathrm{HC}$ at baseline

- $\quad$ SFF was assessed using the LDI $\mathrm{FLARE}_{\mathrm{F}}$ technique, which measured the axon mediated vascular reflex in the foot skin. SFS was assessed using in-vitro corneal confocal microscopy (IVCCM) measuring nerve fibre density (CNFD); branch density (CNBD) and fibre length (CNFL)

- Large fibre neuropathy was assessed by determining sural nerve conduction velocity (SNCV) and amplitude (SNAP) and vibration perception threshold (VPT)

- After optimal replacement of HypoT patients with LT4 (target TSH 2-3 miu/L) for at least 6 months, all subjects including HC's were reevaluated to determine change in both small and large fibre modalities

- All participants underwent biochemical investigations including fasting $\mathrm{TG}, \mathrm{HbA}_{1 \mathrm{c}}$ and routine anthropometry

- Statistical analysis was done using SPSS version 20 for windows
Methods to study small fibre function (LDI $\mathrm{FLARE}_{\text {) }}$ and function (CCM)

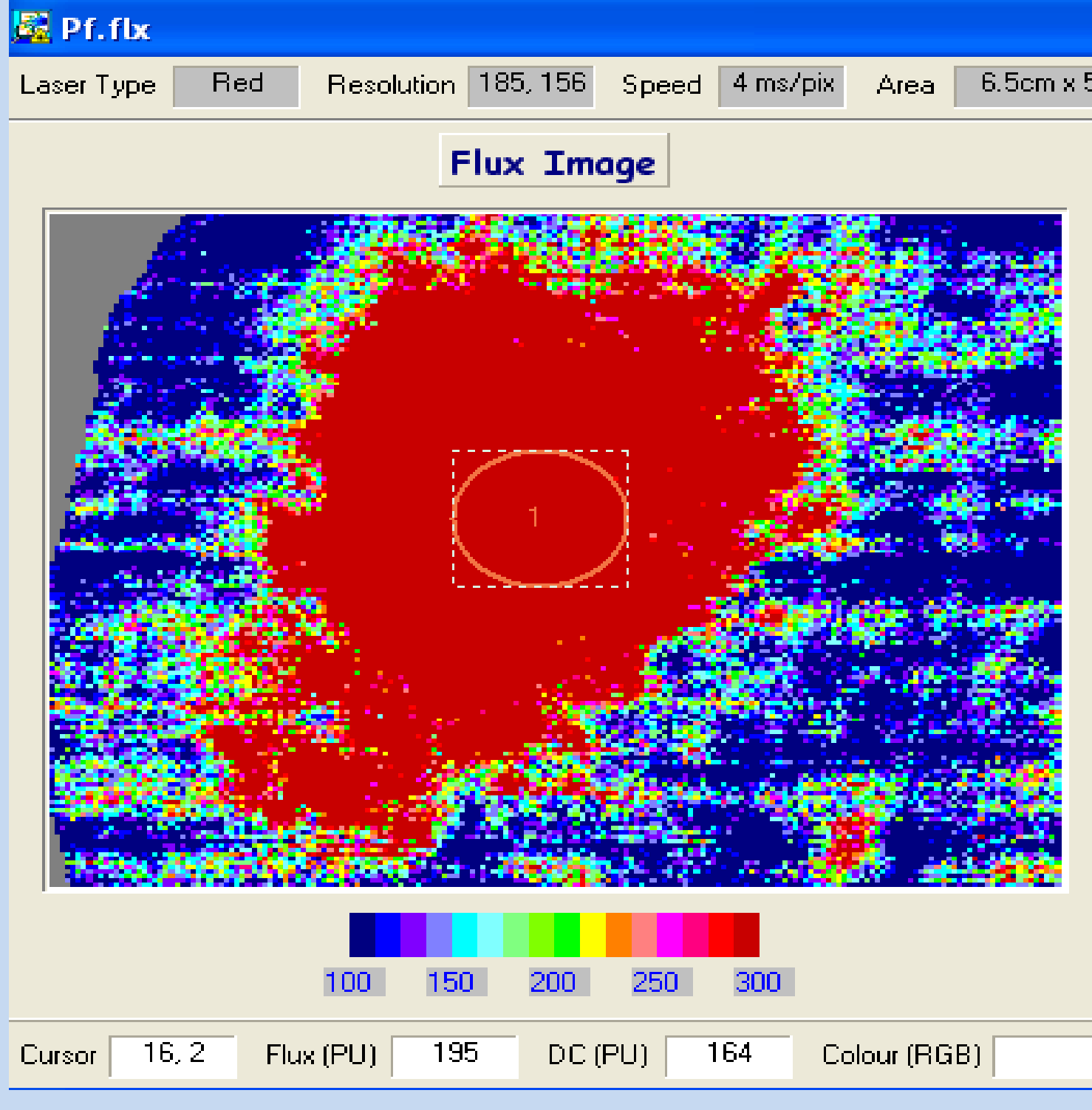

Fig. 1a: LDI $_{\text {FLARE }}$ in HC

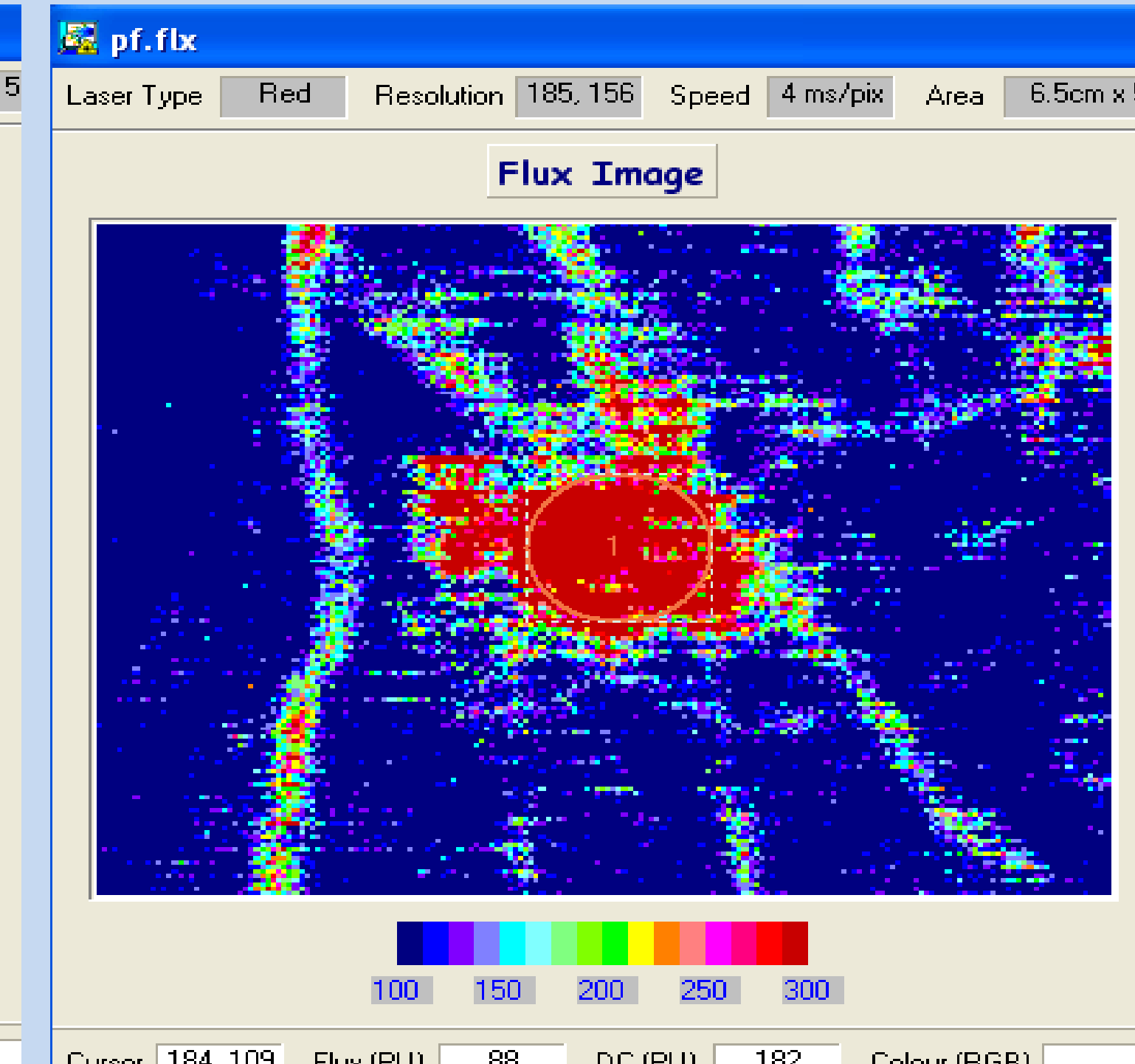

Fig. 1b: LDI FLARE $_{\text {in HypoT }}$

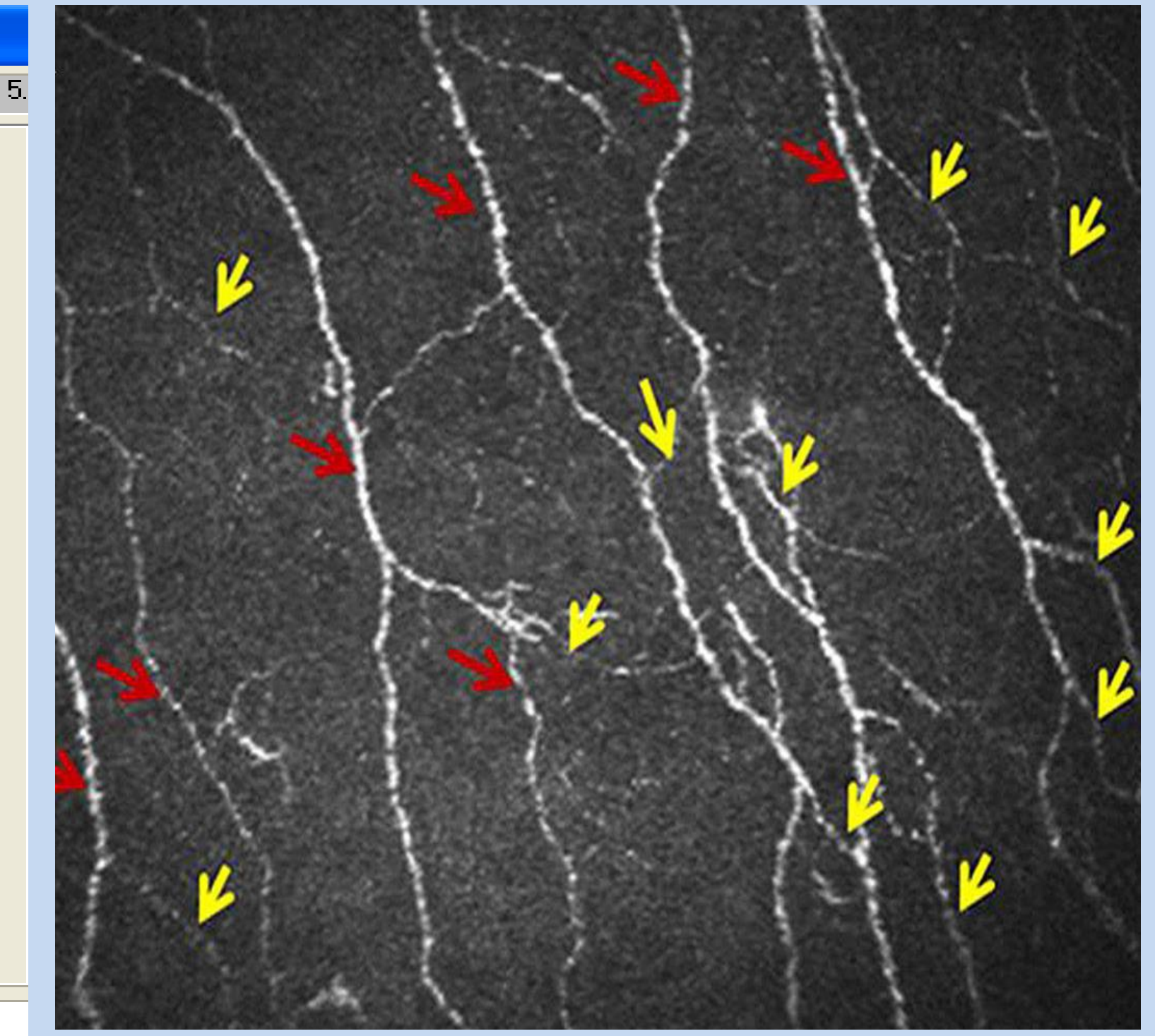

Fig. 2: CNFD assessed by CCM
Results

- Clinical and anthropometric assessments of study participants are shown in Table 1

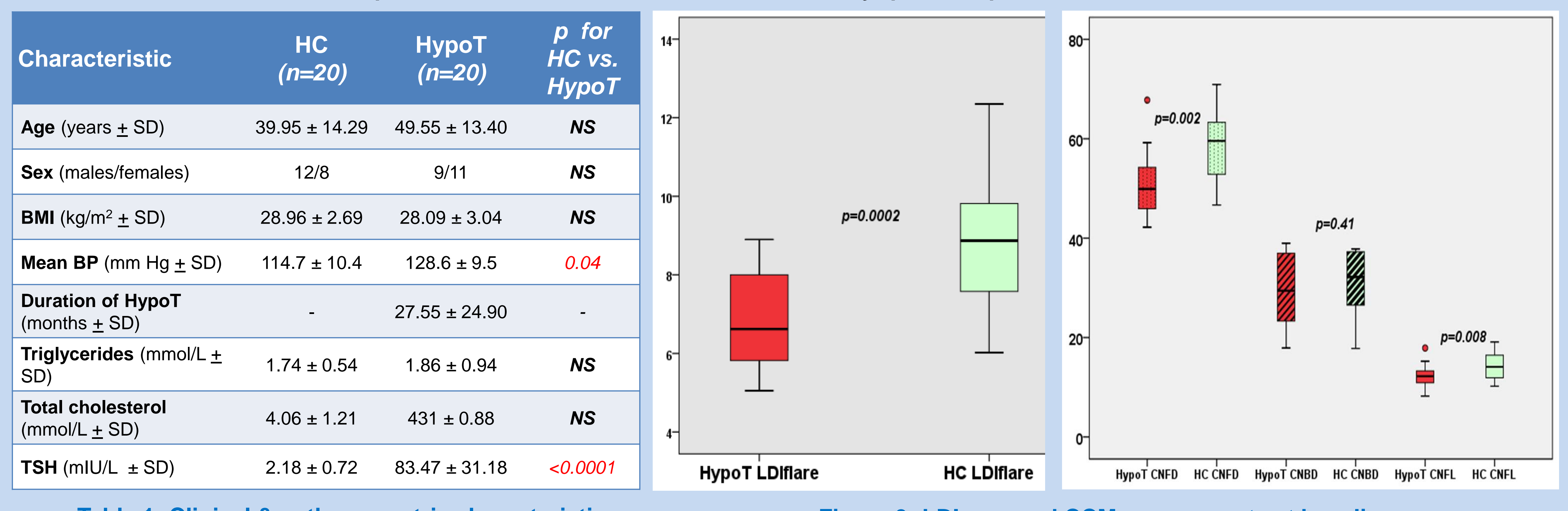

Table 1: Clinical \& anthropometric characteristics

Figure 3: LDI $_{\text {FLARE }}$ and CCM assessments at baseline

- At baseline (Figure 3) $\mathrm{LDI}_{\mathrm{FLARE}}( \pm \mathrm{SD}$ ) was significantly reduced in HypoT compared to $\mathrm{HC}(6.74 \pm 1.20$ vs. $8.90 \pm 1.75 \mathrm{~cm} 2 ; \mathrm{p}=0.0002)$. Of the IVCCM parameters, both CNFD (50.77 \pm 6.54 vs. $58.32 \pm 6.54 \mathrm{no} / \mathrm{mm} 2 ; \mathrm{p}=0.002)$ and CNFL (12.11 \pm 5.45 vs. $14.24 \pm 6.27$ $\mathrm{p}=0.0008)$ were reduced when compared to HC but not CNBD $(p=0.41)$

- Neither SNCV nor SNAP as well as VPT were different compared with HC $(p=0.10$, $p=0.05$ and $p=0.19$ respectively)

- Following LT4 treatment, both LDI $\mathrm{FLARE}_{\text {( }}(7.72 \pm 1.12$ vs. $6.74 \pm 1.20 \mathrm{~cm} 2 ; \mathrm{p}=<0.0001$; Figure 4) and CNFD (54.43 \pm 5.70 vs. $50.77 \pm 6.54 \mathrm{no} / \mathrm{mm} 2 ; \mathrm{p}=0.02)$ improved significantly but neither CNBD nor CNFL changed ( $p=0.26$ and $p=0.06$ respectively; Figure 5)

- Similarly, no significant change was seen with SNCV, SNAP and VPT following treatment. $(p=0.70, p=0.10$ and $p-0.41$ respectively)

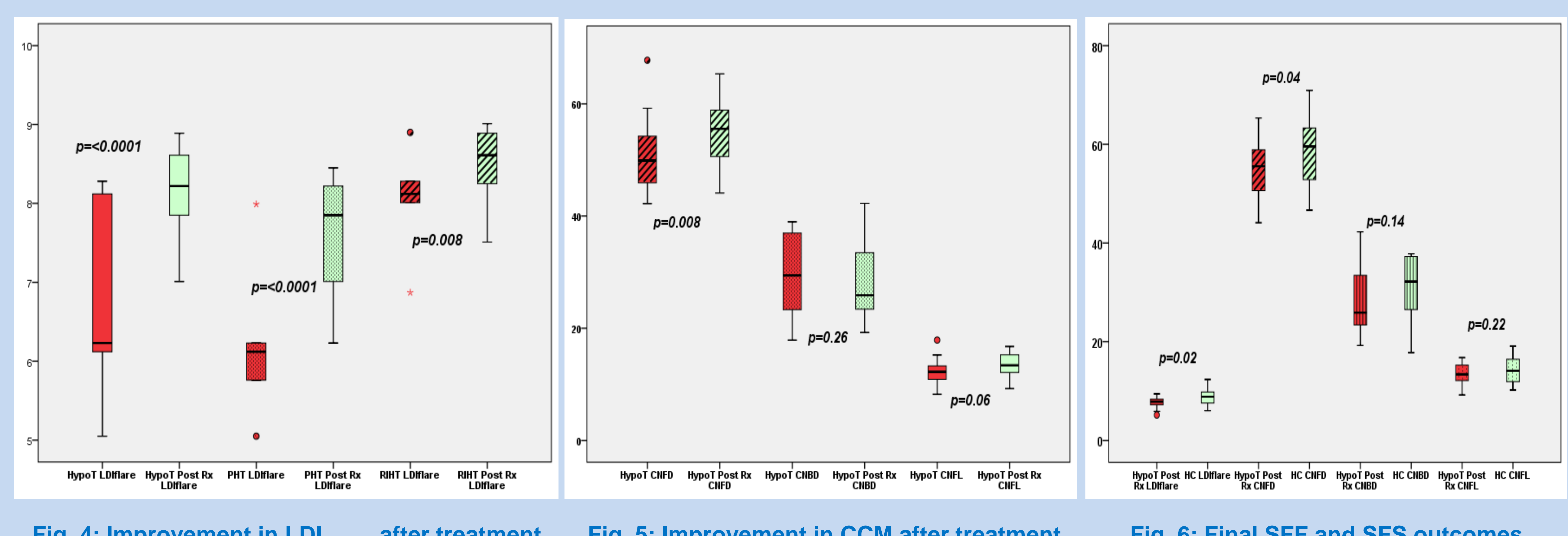

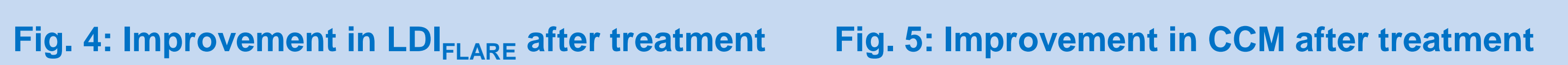

- Interestingly, both small fibre function (LDI ${ }_{\mathrm{FLARE}}$ ) and structure (CCM-CNFD) despite improving in HypoT remained significantly reduced compared to $\mathrm{HC}$ (7.72 \pm 1.12 vs. $8.85 \pm 1.33 \mathrm{~cm} 2 ; p=0.008$ and $54.43 \pm 5.70$ vs. $58.03 \pm 7.98 \mathrm{no} / \mathrm{mm} 2 ; p=0.01$ respectively) at the end of the study period (Figure 6)

\section{Conclusions}

- Our study shows that both small fibre function (assessed by LDI FLARE$_{\text {) }}$ and structure (assessed by CCM) are significantly reduced in untreated HypoT compared to HC while no such difference was seen with large fibre modalities

- After optimal replacement therapy with LT4, the former improved significantly but compared to HC remained low. The latter perhaps suggests that using TSH as the sole biomarker to monitor replacement strategies may not be adequate to improve small fibre dysfunction

- Further studies are required to explore this hypothesis further 